\title{
Time structure of fast domain wall creation by localized fields in a magnetic nanowire
}

Lars Bocklage, Falk-Ulrich Stein, Michael Martens, Toru Matsuyama, and Guido Meier

Citation: Appl. Phys. Lett. 103, 092406 (2013); doi: 10.1063/1.4819729

View online: https://doi.org/10.1063/1.4819729

View Table of Contents: http://aip.scitation.org/toc/apl/103/9

Published by the American Institute of Physics

\section{Articles you may be interested in}

Tunable eigenmodes of coupled magnetic vortex oscillators

Applied Physics Letters 104, 182405 (2014); 10.1063/1.4875618

Local modification of the magnetic vortex-core velocity by gallium implantation

Journal of Applied Physics 115, 103909 (2014); 10.1063/1.4868222

Chemical reactions of conformationally selected 3-aminophenol molecules in a beam with Coulomb-crystallized

$\mathrm{Ca}^{+}$ions

The Journal of Chemical Physics 140, 124202 (2014); 10.1063/1.4869100

Hydration shell effects in the relaxation dynamics of photoexcited Fe-ll complexes in water

The Journal of Chemical Physics 141, 044304 (2014); 10.1063/1.4890528

Coherent or hopping like energy transfer in the chlorosome?

AIP Conference Proceedings 1610, 135 (2014); 10.1063/1.4893524

Fast generation of domain walls with defined chirality in nanowires

Applied Physics Letters 104, 172404 (2014); 10.1063/1.4874803

\section{PHYSICS TODAY}

MANAGER'S GUIDE

WHITEPAPERS
Accelerate R\&D with

Multiphysics Simulation

\section{READ NOW}

PRESENTED BY

И๐ $\subset$ MSOL 


\title{
Time structure of fast domain wall creation by localized fields in a magnetic nanowire
}

\author{
Lars Bocklage, ${ }^{1,2,3, a)}$ Falk-Ulrich Stein, ${ }^{2}$ Michael Martens, ${ }^{2}$ Toru Matsuyama, ${ }^{2}$ \\ and Guido Meier ${ }^{2,3}$ \\ ${ }^{1}$ Deutsches Elektronen-Synchrotron DESY, Notkestraße 85, 22607 Hamburg, Germany \\ ${ }^{2}$ Institut für Angewandte Physik und Zentrum für Mikrostrukturforschung, Universität Hamburg, \\ Jungiusstrasse 11, 20355 Hamburg, Germany \\ ${ }^{3}$ The Hamburg Centre for Ultrafast Imaging, Luruper Chaussee 149, 22761 Hamburg, Germany
}

(Received 15 July 2013; accepted 14 August 2013; published online 27 August 2013)

\begin{abstract}
We have investigated the generation of magnetic domain walls by nanosecond magnetic field pulses of a strip line. Domain wall creation is sensitive to an externally applied field concerning wall type and threshold amplitudes. The domain wall creation is stochastic similar to domain wall depinning. In the experiment reliable domain wall generation require up to $8 \mathrm{~ns}$ long pulses at the threshold field amplitude. The required pulse length can be reduced by higher field amplitudes. Time-resolved measurements and micromagnetic simulations show that the domain wall is generated within three nanoseconds. The creation proceeds via vortex core formation under the strip line. ㅇ 2013 AIP Publishing LLC. [http://dx.doi.org/10.1063/1.4819729]
\end{abstract}

Magnetic domain walls in nanowires have been proposed for use in logic devices and magnetic memories. ${ }^{1,2}$ The current-induced motion of domain walls along nanowires was investigated intensively. ${ }^{3-6}$ For technical application, the creation of domain walls on a nanosecond timescale is essential. Most studies on domain walls use external magnetic fields to create a domain wall in a nanowire. This procedure is slow, acts globally, and is therefore not suitable for devices processing many domain walls. Another approach is given by a localized switching of the magnetization by the magnetic Oersted field that accompanies a current running through a strip line. ${ }^{7}$ This localized creation is efficient and reliable and has been used in studies on domain wall motion in nanowires, ${ }^{2,8-10}$ but only few investigations have analyzed this process. ${ }^{11-14}$ Time-resolved X-ray microscopy shows that the domain wall emerges within a few nanoseconds, ${ }^{15}$ but the technique is not suited to study phase spaces of parameters due to limited availability. We use quasi-static and time-resolved magnetoresistance measurements as well as micromagnetic simulations to investigate the nucleation progress of the domain walls.

Figure 1(a) shows a scanning electron micrograph of a permalloy $\left(\mathrm{Ni}_{80} \mathrm{Fe}_{20}\right)$ nanowire. Nanowires are prepared on a silicon substrate by electron-beam lithography, thermal evaporation, and lift-off processing. The wires are $8 \mu \mathrm{m}$ long, have a width in the range of 215 and $230 \mathrm{~nm}$, and a thickness of $20 \mathrm{~nm}$. Wave guides are fabricated via a second lithography step to contact the wire. Before d.c. magnetron sputtering of $3 \mathrm{~nm}$ aluminum and $5 \mathrm{~nm}$ gold, the wires are cleaned by in-situ Argon plasma etching to ensure ohmic contacts. Subsequently, $90 \mathrm{~nm}$ copper and $5 \mathrm{~nm}$ gold are deposited by thermal evaporation. The samples are contacted by probes and cables with a bandwidth of $20 \mathrm{GHz}$. Direct currents of $100 \mu \mathrm{A}$ are fed into the high-frequency setup via bias tees for resistance measurements of the permalloy wires. The presence of a domain wall is determined from the

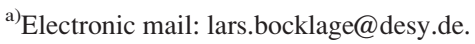

anisotropic magnetoresistance before and after the pulse. ${ }^{16}$ Every set of parameters is measured ten times. The Oersted field generated by a voltage pulse is calculated by $\mu_{0} H_{p}=\mu_{0} I /(2 w),{ }^{17}$ where $w=550 \mathrm{~nm}$ is the width of the strip line and $I=V_{\text {osc }} / 50 \Omega$ is the current through the strip line calculated from the voltage drop $V_{\text {osc }}$ measured with an oscilloscope terminating the strip line.

Before the generation of domain walls, the wire is saturated along the $x$-direction in an external field of $100 \mathrm{mT}$. A voltage pulse through the right strip line is applied to generate an Oersted field pointing in negative $x$-direction (red) that switches the initial magnetization (blue) in the region below the strip line (yellow). On either side of the strip line a domain wall emerges. By repetitive measurements of the wire resistance before and after the pulse the probability for the domainwall generation between the contacts is determined.

The external field and pulse amplitudes necessary for the formation of domain walls are investigated prior to the time dependent measurements. A probability plot for varying pulse amplitudes and external magnetic fields is given in Fig. 1(b). The pulse length is $10 \mathrm{~ns}$ with rise times of $2 \mathrm{~ns}$. We identify four regions (I-IV) separated by the dotted black lines. In region I, no domain walls are generated because the Oersted field $\mu_{0} H_{p}$ is too low. Region III has a high probability for domain wall generation between $-1.6 \mathrm{mT}$ and $1 \mathrm{mT}$ and for pulse voltages below a threshold of $-2.6 \mathrm{~V}$. A shift of the threshold voltage proportional to the external field is measured. This shift is understood as the external field alters the total applied field $H_{t o t}=H_{O e}+H_{e x t}$ and results in a lower or higher pulse amplitude necessary for domain wall generation. The slope of the region I boundary is given by $a$ $=-18.58 \mathrm{mT} / \mathrm{V}$. By a linear correlation of the voltage $V$ and the $x$-components of Oersted field pulse $\mu_{0} H_{p}$ we get $\mu_{0} H_{p}=a V$. This offers a straightforward way to determine the Oersted field amplitude from the applied voltage without measuring the actual voltage drop and results in the same field values as the one calculated from Ref. 17. In region II and IV domain walls are generated but the external field is 
(a)

(b)
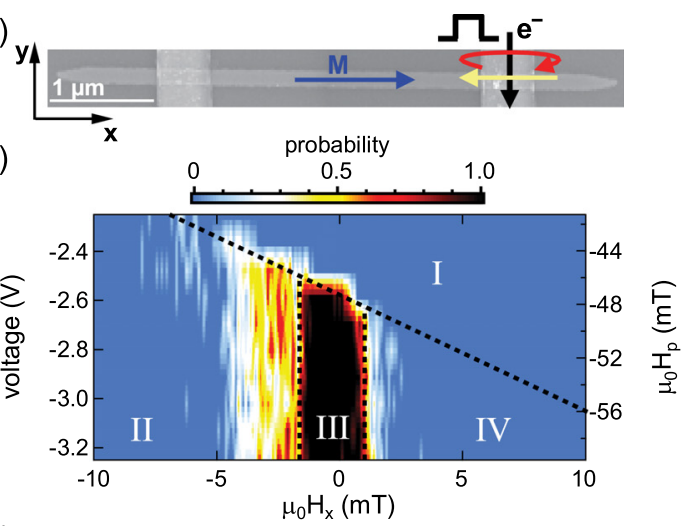

(c)

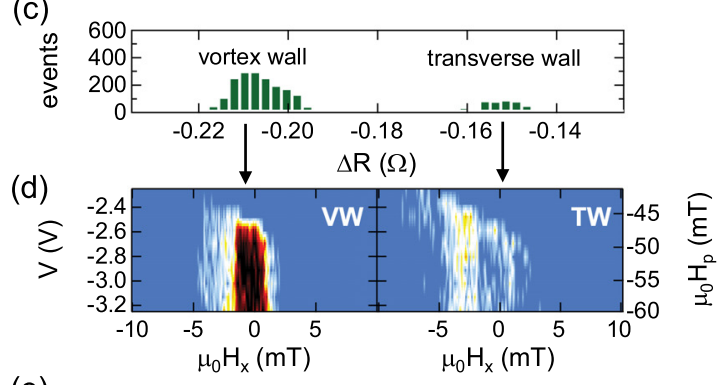

(e)

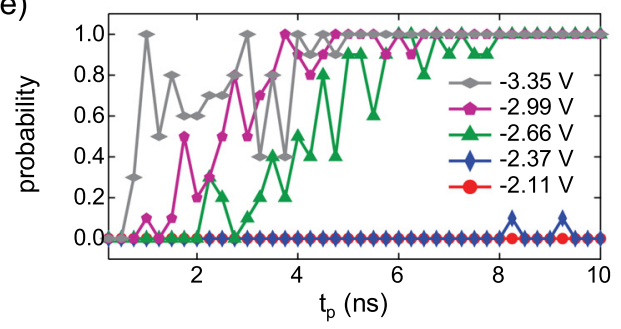

FIG. 1. (a) Scanning electron micrograph of a permalloy nanowire with two contact lines and a scheme of the domain wall generation. (b) Probability plot of the domain wall generation depending on the external field $\mu_{0} H_{x}$ along the wire and on the pulse amplitude. The $x$-component of the Oersted field $\mu_{0} H_{p}$ is calculated from the applied voltage. Pulse length and rise time are $10 \mathrm{~ns}$ and $2 \mathrm{~ns}$, respectively. (c) Histogram of the resistance changes due to the domain wall measured in (a). (d) Probability plots of the generation of vortex walls (VW) and transverse walls (TW). (e) Probability of domain wall generation in dependence of the voltage pulse length $t_{p}$ for different pulse amplitudes in zero external field. The rise time of the pulses is $65 \mathrm{ps}$.

larger than the propagation field leading to domain wall motion and annihilation at the ends of the wire (II) or motion, collision, and mutual annihilation (IV) depending on the external field direction. Due to the different resistance changes of vortex and transverse domain walls, we can distinguish the generated domain wall type. ${ }^{18}$ Figure $1(\mathrm{c})$ shows a histogram of the resistance changes corresponding to vortex and transverse domain walls. The probability plots for each wall type (see Fig. 1(d)) demonstrate that mainly vortex walls are generated. Transverse walls are predominantly generated at negative fields below $-1.6 \mathrm{mT}$. From $-1.6 \mathrm{mT}$ to $-5 \mathrm{mT}$, the probability is low for both wall types.

These measurements show that the creation of domain walls is stochastic similar to the depinning of domain walls. ${ }^{19}$ In region III where the generation is $100 \%$ reliable the generation is stochastic because a non-vanishing probability to generate transverse walls remains. However, for some parameters, we get a deterministic generation of vortex walls (although the 10 repetitions are quite low) as shown in Fig. 1(d).
The pulse length and the pulse amplitude are varied in zero field, where almost exclusively vortex domain walls are created. Figure 1(e) shows the measured probabilities for domain wall generation for different pulse lengths and amplitudes. Here, the rise time of the pulse generator is $65 \mathrm{ps}$. When the threshold voltage of $-2.6 \mathrm{~V}$ is reached, the probability for domain wall nucleation increases. With increasing pulse amplitude, the required pulse length is reduced from $4 \mathrm{~ns}$ at $-2.66 \mathrm{~V}$ to $1 \mathrm{~ns}$ at $-3.35 \mathrm{~V}$ for a $50 \%$ generation probability. Reliable domain wall generation is achieved for times longer than $8 \mathrm{~ns}$ for all pulse amplitudes above the threshold voltage. Amplitudes explicitly exceeding the threshold voltage $(-2.99 \mathrm{~V}$ and $-3.35 \mathrm{~V})$ result in reliable generation at about $4 \mathrm{~ns}$.

The experimental results are compared to micromagnetic simulations. First, we calculate the Oersted field distribution around a strip line numerically by integrating Biot-Savarts law for a given current density. The simulated strip line has a width of $550 \mathrm{~nm}$ and a thickness of $100 \mathrm{~nm}$, being close to the parameters from the experiments. The calculated Oersted field amplitude has the same time-development as the rectangular voltage pulse characterized by the pulse width that is the full width at half maximum and the rise and fall times. ${ }^{20}$ The rise and fall times are set to the values of the two electrical pulse generators of either $2 \mathrm{~ns}$ or 65 ps used in the experiment. Micromagnetic simulations are performed with the Object Oriented MicroMagnetic Framework (OOMMF). ${ }^{21}$ The exchange constant $A=1.3 \times 10^{11} \mathrm{~J} / \mathrm{m}$, the saturation magnetization $M_{s}=860 \mathrm{kA} / \mathrm{m}$, and the Gilbert damping parameter $\alpha=0.01$ are used as material parameters for permalloy. The size of the simulation cells is $5 \mathrm{~nm} \times 5 \mathrm{~nm} \times 20 \mathrm{~nm}$. The simulated wires are $4 \mu \mathrm{m}$ long, $230 \mathrm{~nm}$ wide and have a edge roughness of $5 \mathrm{~nm}$ to introduce small pinning. The center of the strip line is located $1 \mu \mathrm{m}$ away from the right side of the wire. Figure 2 shows results of the simulations. The four different regions indicated in the experimental data are found in the simulations shown in Fig. 2(a). The events in green and grey correspond to the formation of vortex walls. Green events around zero field are vortex walls that remain in the wire, while grey event are vortex walls that propagate through the wire. Transverse walls are shown in yellow and are more likely produced at elevated external field amplitudes in agreement with the experimentally observed occurrence of transverse walls mainly at negative fields below $-1.6 \mathrm{mT}$.

The exact sequence of domain wall nucleation depends on the wire and stripline geometry. Exemplarily we discuss the mechanism in our measured and simulated geometry for a current density of $1.2 \times 10^{11} \mathrm{~A} / \mathrm{m}^{2}$ in zero field with a pulse duration of $10 \mathrm{~ns}$ and a risetime of $2 \mathrm{~ns}$ where vortex walls are generated. The nucleation is shown in Fig. 2(c) at different time steps after the pulse starts. The process is initiated by an opposed rotation of the magnetization under the strip line $(2.59 \mathrm{~ns})$ resulting in the formation of a vortex core at the lower edge that propagates across the wire width $(2.94 \mathrm{~ns})$. Two transverse walls originate when the vortex core hits the upper edge of the wire $(3.40 \mathrm{~ns})$. The transverse walls transform shortly after their creation into vortex walls by vortex core nucleation at the wire edges $(3.98 \mathrm{~ns})$. For the events where transverse walls are generated the vortex cores are annihilated at the wire edges. The vortex core deflection to the 
(a)

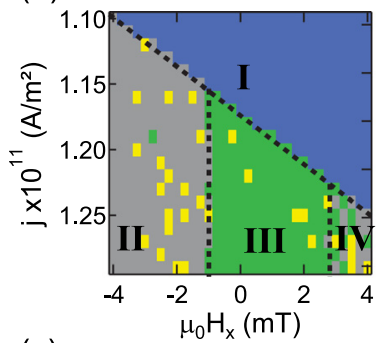

(c)

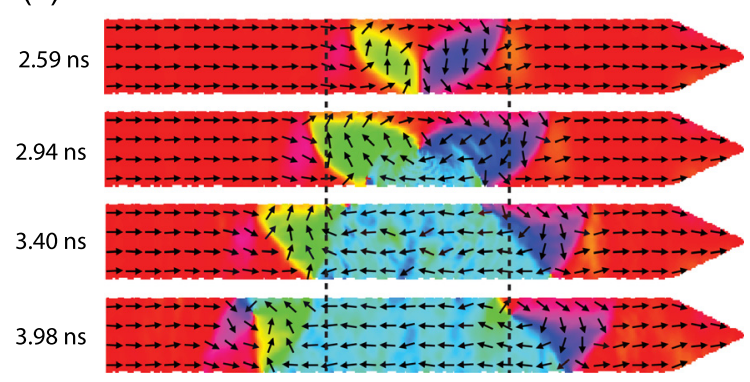

FIG. 2. Results of micromagnetic simulations of domain wall generation. (a) Simulated processes of domain wall generation in dependence on the current density $j$ through the strip line and the external field $\mu_{0} H_{x}$ along the wire. The Oersted field $\mu_{0} H_{p}$ is the peak value of the $x$-component during the $10 \mathrm{~ns}$ long pulse with a rise time of $2 \mathrm{~ns}$. The wire is $230 \mathrm{~nm}$ wide and $20 \mathrm{~nm}$ thick. (Blue) No domain wall generation, (green) generation of a vortex wall, (yellow) generation of a transverse wall, (grey) generation, propagation, and subsequent annihilation of a vortex wall. (b) Simulated processes of domain wall generation in dependence on the current density $j$ through the strip line and the pulse length $t_{p}$ in zero field with rise times of $65 \mathrm{ps}$. Red indicates that domain walls are generated and subsequently annihilate each other. (c) Simulations of domain wall generation at various times after the pulse is applied. The simulation is performed in zero external field with a current density of $1.2 \times 10^{11} \mathrm{~A} / \mathrm{m}^{2}$, a pulse duration of $10 \mathrm{~ns}$, and a risetime of $2 \mathrm{~ns}$. The dashed lines indicate the edges of the strip line.

edges scales with the external field and the vortex annihilation at the edge takes place at elevated external field amplitudes. ${ }^{22}$ Due to the transformation and the higher depinning fields of the transverse walls, we observe mainly transverse walls in the experiments and in the simulations in regions II and IV.

Simulations depending on the pulse length are shown in Fig. 2(b). Domain walls are observed starting at a threshold current density of $1.18 \times 10^{11} \mathrm{~A} / \mathrm{m}^{2}(64 \mathrm{mT})$. For current densities close to the threshold simulations show that the domain wall creation starts at pulse length of $2.5 \mathrm{~ns}$. Slightly shorter pulse lengths lead to the creation of two vortex walls but the pulse length is too short to separate the walls from each other leading to their mutual annihilation by attractive interaction (red). For current densities exceeding the threshold field the required pulse length reduce to $1.5 \mathrm{~ns}$. Simulations in Ref. 12 showed similar time scales for domain wall generation. Comparison of our simulations with the measurements shown in Fig. 1(e) evidences a very good agreement concerning the minimum time scales for domain wall generation. The stochastic nature is of course not reproduced by the simulations.

Quasi-static measurements can only detect domain walls after the pulse is applied. For the study of the progress of domain wall creation time-resolved magnetoresistance measurements are performed. These measurements are carried out on a different wire than the measurements discussed above. The overall behavior and the created wall types are very similar to the measurements before but with slightly higher propagation fields and threshold voltages as shown in Figs. 3(a) and 3(b). These time-resolved measurements are performed with a real-time oscilloscope with a bandwidth of $4 \mathrm{GHz}$. Considering the pulse length dependence shown in Fig. 1(e) that give a reliable generation at 8 ns we choose a pulse duration of $10 \mathrm{~ns}$ to ensure domain wall creation. The rise time is $65 \mathrm{ps}$ and measurements are carried out in zero external field for preceding saturation fields of $100 \mathrm{mT}$ or $-100 \mathrm{mT}$. For a negative saturation field, no domain wall is created as the Oersted field and the magnetization are aligned parallel. The differences of the voltage traces with opposite preceding fields are averaged 7500 times to increase the signal-to-noise ratio. A pulse amplitude of $-3.4 \mathrm{~V}$ close to the threshold voltage is used to create the domain walls.

Time-resolved measurements of the anisotropic magnetoresistance are shown in Fig. 3(c). The voltage drop immediately changes as the pulse is applied (within the rise time of $65 \mathrm{ps}$ ). The voltage drop increases until about $2.7 \mathrm{~ns}$ where the plateau value is reached, indicating that the vortex wall has fully emerged between the contacts. The voltage drop then changes for a few nanoseconds due to alterations in the domain-wall structure. These structural changes have been observed by time-resolved X-ray microscopy. ${ }^{15}$ The time scale of $2.7 \mathrm{~ns}$ fits perfectly to the X-ray microscopy data ${ }^{15}$ and quite well to simulations we performed with a rise time of $65 \mathrm{ps}$ where a duration of $2 \mathrm{~ns}$ is determined (not shown).

In summary, we have determined the Oersted field strength to generate domain walls with a strip line aligned

(a)
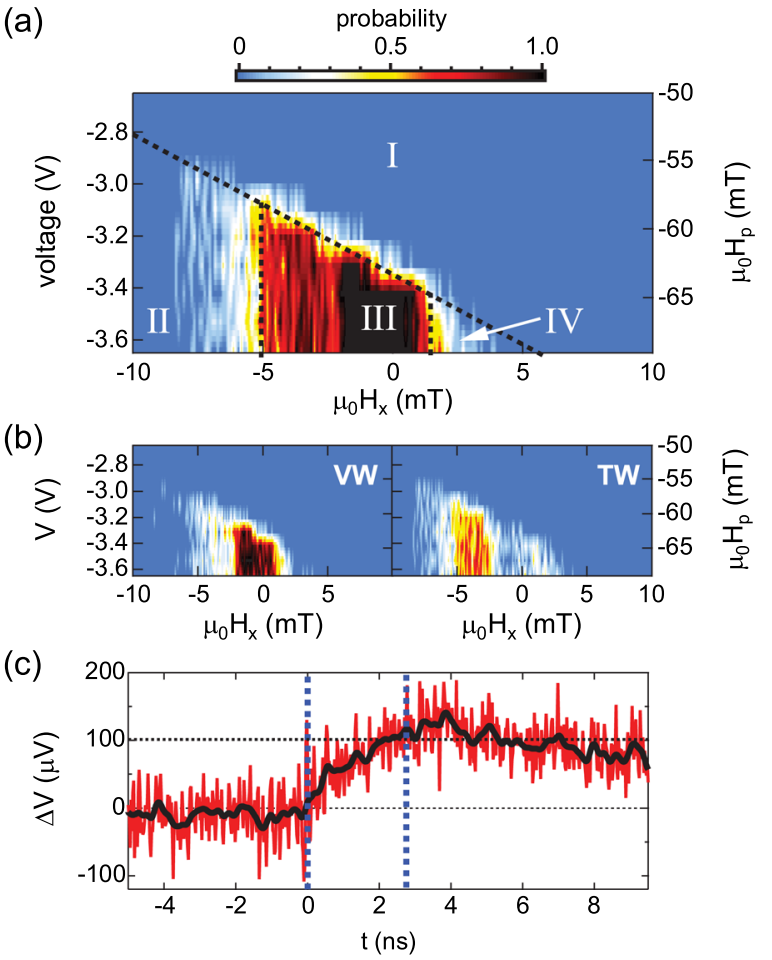

FIG. 3. Experimental results on domain wall generation for the wire of the time-resolved measurements. (a) Probability plot of the domain wall generation depending on the external field $\mu_{0} H_{x}$ along the wire and on the pulse amplitude. The $x$-component of the Oersted field $\mu_{0} H_{p}$ is calculated from the slope of region I. Pulse length and rise time are $10 \mathrm{~ns}$ and $2 \mathrm{~ns}$, respectively. (b) Probability plots of the generation of vortex walls (VW) and transverse walls (TW). (c) Time-resolved anisotropic magnetoresistance measurement in the wire (red) and smoothed data (black). The rise time of the pulse is $65 \mathrm{ps}$. 
perpendicular to a nanowire. The created domain wall types are not only given by the wire dimensions but also by the external field that as well changes the minimum voltage amplitudes. The generation process is stochastic but external parameters can be found where the process gets deterministic. A generation time of about $2.7 \mathrm{~ns}$ for the domain wall is determined by time-resolved magnetoresistance measurements. The minimum pulse length to create a domain wall depends on the voltage amplitude. A reliable creation is achieved for pulse lengths of $8 \mathrm{~ns}$ for all pulse amplitudes above the threshold. In the measured wires with dimensions of technological relevance the nucleation proceeds via an opposed rotation of the magnetization under the strip line and the formation of a vortex core at one edge which subsequently initiates the formation of two domain walls by a cross propagation through the wire.

We thank Ulrich Merkt and Thomas Kamionka for fruitful discussions and Michael Volkmann for excellent technical support. Financial support of the Deutsche Forschungsgemeinschaft via the Sonderforschungsbereich 668 and via the Graduiertenkolleg 1286 is gratefully acknowledged.

${ }^{1}$ D. A. Allwood, G. Xiong, C. C. Faulkner, D. Atkinson, D. Petit, and R. P. Cowburn, Science 309, 1688 (2005).

${ }^{2}$ M. Hayashi, L. Thomas, R. Moriya, C. Rettner, and S. S. P. Parkin, Science 320, 209 (2008).

${ }^{3}$ A. Yamaguchi, T. Ono, S. Nasu, K. Miyake, K. Mibu, and T. Shinjo, Phys. Rev. Lett. 92, 077205 (2004).
${ }^{4}$ S. S. P. Parkin, M. Hayashi, and L. Thomas, Science 320, 190 (2008).

${ }^{5}$ G. Meier, M. Bolte, R. Eiselt, B. Krüger, D.-H. Kim, and P. Fischer, Phys. Rev. Lett. 98, 187202 (2007).

${ }^{6}$ M. Kläui, P.-O. Jubert, R. Allenspach, A. Bischof, J. A. C. Bland, G. Faini, U. Rüdiger, C. A. F. Vaz, L. Vila, and C. Vouille, Phys. Rev. Lett. 95, 026601 (2005).

${ }^{7}$ M. Hayashi, L. Thomas, Y. B. Bazaliy, C. Rettner, R. Moriya, X. Jiang, and S. S. P. Parkin, Phys. Rev. Lett. 96, 197207 (2006).

${ }^{8}$ M. Hayashi, L. Thomas, C. Rettner, R. Moriya, and S. S. P. Parkin, Nat. Phys. 3, 21 (2007).

${ }^{9}$ L. Thomas, R. Moriya, C. Rettner, and S. Parkin, Science 330, 1810 (2010).

${ }^{10}$ M. Muñoz and J. L. Prieto, Nat. Comm. 2, 562 (2011).

${ }^{11}$ M. Hayashi, L. Thomas, C. Rettner, R. Moriya, Y. B. Bazaliy, and S. S. P. Parkin, Phys. Rev. Lett. 98, 037204 (2007).

${ }^{12}$ J. L. Prieto, M. Muñoz, and E. Martínez, Phys. Rev. B 83, 104425 (2011).

${ }^{13}$ F.-U. Stein, L. Bocklage, T. Matsuyama, and G. Meier, Appl. Phys. Lett. 100, 192403 (2012).

${ }^{14}$ L. O’Brien, D. E. Read, D. Petit, and R. P. Cowburn, J. Phys.: Condens. Matter 24, 024222 (2012).

${ }^{15}$ F.-U. Stein, L. Bocklage, M. Weigand, and G. Meier, Sci. Rep. 3, 1737 (2013).

${ }^{16} \mathrm{G}$. Nahrwold, L. Bocklage, J. M. Scholtyssek, T. Matsuyama, B. Krüger, U. Merkt, and G. Meier, J. Appl. Phys. 105, 07D511 (2009).

${ }^{17}$ T. J. Silva, C. S. Lee, T. M. Crawford, and C. T. Rogers, J. Appl. Phys. 85, 7849 (1999).

${ }^{18}$ M. Hayashi, L. Thomas, C. Rettner, R. Moriya, X. Jiang, and S. S. P. Parkin, Phys. Rev. Lett. 97, 207205 (2006).

${ }^{19}$ M.-Y. Im, L. Bocklage, P. Fischer, and G. Meier, Phys. Rev. Lett. 102, 147204 (2009).

${ }^{20}$ L. Bocklage, B. Krüger, T. Matsuyama, M. Bolte, U. Merkt, D. Pfannkuche, and G. Meier, Phys. Rev. Lett. 103, 197204 (2009).

${ }^{21} \mathrm{M}$. Donahue and D. Porter, OOMMF User's Guide, Version 1.0, Interagency Report NISTIR 6376 (National Institute of Standards and Technology, Gaithersburg, MD, 1999).

${ }^{22}$ D. J. Clarke, O. A. Tretiakov, G.-W. Chern, Y. B. Bazaliy, and O. Tchernyshyov, Phys. Rev. B 78, 134412 (2008). 